\title{
The Correlation of Complex Defensive Movements between Playing Positions in the Formation (5:1) Used by the French National Team, as a Guide for Tactical Training of Beginners. Analytical Study Based on the World Handball Championship - Croatia 2009.
}

\author{
Mohamed Ashraf Mohamed Kamel*
}

The analytical study of handball matches played by international teams in the Croatia World Championship of 2009 was conducted by applying an analysis form designed by the researcher. This form was subjected to statistical treatment, to ensure its suitability for analysis, and to identify the complex defensive movements in the formation (5:1), as a major defensive formation widely used in the mentioned world championship, by listing the types of complex defensive movements through individual tactical performance from the various playing positions, and the effect of defensive movements during defense on the tactical formations of One-toone marking, and delivery and receiving.

The results of the analysis of the basic study sample teams show the great role of defensive movements of the positions of the center and back players. This is compatible with the results of the International Handball Federation concerning the performance analysis of the French team after ten matches, and it is also confirmed by the correlation between the movements of the two midfielders and the two backs, that was significant at the level (0.01), thus reflecting the burden that these positions should bear to face the offense, as $85 \%$ of the scoring attempts came from the midfield.

The results of the analysis of individual tactical performance (of the French team) show the importance of the midfield players in the (5:1) formation through the closed-defense method, as they are responsible for deciding the direction of defensive performance during organized defense. The outcomes, also, confirmed the importance of the harmony between motor duties of the adjacent cooperating positions in the defense line through One-to-one marking and delivery and receiving, that can be considered as a mainstay of guiding tactical training, especially for beginners, where simulating the actual game performance process adds an important dimension to the effectiveness of the preparation of tactics .

Key Words: complex defensive movements- defense formation (5:1) - analytical study- world handball championship, Croatia 2009- guiding tactical training of beginners.

\section{Introduction:}

$\mathrm{H}$ andball experts in Egypt and elsewhere, Darwish et al, (1998), Pollany (2000),Feldman (2003) agree that group defense depends on the players' ability to perform their defensive duties.

The role of the defender is not only to keep an eye on a certain player according to the method of playing,

\footnotetext{
*Lecturer, Sports Games Training Department, Faculty of Sport Education, Alexandria University, National Coach, registered with the Egyptian Handball Federation and Former International Player in the Egyptian National Team of Handball.
}

but also to help the defender and cover him all the time. However clever the defender may be, he cannot be excellent alone or without his team members in the defense line beside him who move in harmony with him. Klein (1988), Taborsky (1999), Dios (2000) and Molino (2003) confirmed this fact, where upgrading the proficiency level of the defensive group work relies on the coordination and distribution of the players' role and the defensive requirements. 
The objective observation of the defensive tactical performances in the last world championship (Croatia 2009) showed an extensive use of formation (5:1) in its various methods and styles by most of the participating teams; the high level of quick adaptation to the situations that takes place in the defensive region by confusing the offensive plan of the competitors during disrupting the passes among the play-makers, and by preventing the passes from the play-makers to the player on the centre circle, thus, restricting the danger of back strikers who are skilful at passing and shooting from far places especially in the area facing the goal, and other movements and defensive duties.

There is a correlation between the movements of some positions and others in the defense formation (5:1) by One-to-one marking and delivery and receiving. This compatibility between these movements includes effectiveness in the group work against offense. The study of the success and failures of these movements may give us an evaluation of the defensive ability of the team, and identify the deficiencies in the defensive tactical work, that should be overcome through tactical training.

Accordingly, the study assumes that there is a statistically significant correlation between some complex defensive movements of the midfield player and other defensive positions in the formation $(5: 1)$. This is related to the success or failure of the defensive performance and, consequently, influencing the results of matches. This study aims at reporting the most important mutual significant relations between the types of complex defensive movements of the different playing positions of the French team, who was world champion in 2009, which can be considered as guidelines for the process of tactical training, especially for the beginners' sector of handball.

\section{Research Procedures:}

\section{Research Sample:}

The research sample consists of 14 matches from the world championship of handball, Croatia 2009. These matches were distributed according to table no. 1 in two groups. The first group represents the pilot study ( 8 matches) to decide the axes and items of the forms of the most common complex defensive movements, in the different playing positions, and to find the scientific correlation (validity- reliability and objectivity) for the suggested Form to be used to analyze the matches, while the second group consists of 6 matches in the preliminary and final rounds and include the first four ranked teams in the championship: (FranceCroatia- Poland- Denmark). 
Table (1)

Research Sample (14 matches)

\begin{tabular}{|l|l|l|l|c|c|}
\hline \multicolumn{2}{|c|}{ Pilot Study } & \multicolumn{2}{c|}{ Basic Study } \\
\hline \multicolumn{2}{|c|}{ Form Construction } & Form Scientific Treatment & \multicolumn{2}{c|}{ Analysis of Defensive Movements } \\
\hline \multicolumn{1}{|c|}{ Matches } & Rounds & Matches & Rounds & Matches & Rounds \\
\hline $\begin{array}{l}\text { Tunisia vs } \\
\text { Macedonia }\end{array}$ & Preliminary & $\begin{array}{c}\text { Algeria vs } \\
\text { Macedonia }\end{array}$ & Preliminary & $\begin{array}{c}\text { Croatia vs } \\
\text { Korea }\end{array}$ & Preliminary \\
\hline $\begin{array}{l}\text { Egypt vs } \\
\text { Denmark }\end{array}$ & Preliminary & Kuwait vs Spain & Preliminary & $\begin{array}{c}\text { Spain vs } \\
\text { Croatia }\end{array}$ & Preliminary \\
\hline Egypt vs Russia & Ranking & $\begin{array}{c}\text { Egypt x } \\
\text { Saudi Arabia }\end{array}$ & Preliminary & $\begin{array}{c}\text { France vs } \\
\text { Denmark }\end{array}$ & Semi-final \\
\hline Egypt vs Tunisia & Ranking & Egypt x Serbia & Preliminary & $\begin{array}{c}\text { Croatia } x \\
\text { Poland }\end{array}$ & $\begin{array}{c}\text { Semi-final } \\
\text { Poland }\end{array}$ \\
\hline & & & & $\begin{array}{c}\text { France vs } \\
\text { Croatia }\end{array}$ & $\begin{array}{c}3^{\text {rd }} \text { and } 4^{\text {th }} \\
\text { Positions }\end{array}$ \\
\hline
\end{tabular}

\section{Analyzing the Complex Defensive Movements Analysis Form Construction}

After analyzing the game performance of some of the teams participating in the world championship of Croatia 2009, (Tunisia vs Macedonia), (Egypt vs Denmark), (Egypt vs Russia) and (Egypt vs Tunisia), and consulting specialized references in handball, i.e., researches, studies and training experiences, it was possible to determine the axes and items of the types of complex defensive movements for all playing positions according to the closed defense method in the formation (5:1) as follows:

A - Complex defensive movement types during the individual tactical performance in all playing positions: an attacker - against a defender in all playing positions;

B - Complex defensive movement types during defense in tactical formations (marking One-toone );

- Complex defensive movements types during cutting between two side-by-side players or two players about to be so;
- Complex defensive movements during half of exchanging the positions between two side-by-side players, or two players about to be so.

C - Complex defensive movement types during defense in tactical formations through delivery and receiving.

- Complex defensive movements during defense on exchanging the positions between two side-by-side players, or two players about to be so;

- Complex defensive movements during screening between two side-by-side players, or two players about to be so.

As a second step and in the light of the analytical study, all the complex defensive movements were reported as items for the axes decided in the first step, (figures $a, b$ and c).

\section{Scientific Treatments of the Form:}

- To standardize the form (calculating the scientific treatments of its items), the sample of 
the pilot study matches (Algeria vs Macedonia), (Kuwait vs Spain), (Egypt vs Saudi Arabia) and (Egypt vs Serbia) was selected. Analysis results were used to conduct the needed statistical treatments necessary to calculate the coefficient.

- To calculate the validity of the analysis form content, the suggested form of the Observation Form was referred to handball experts, Hammouda et al. The outcomes showed that the form reflects a high degree of content validity as far as analysis axes are concerned. The Validity degree ranged between $89 \%$ as in the types of complex defensive movements during defense in tactical formations (delivery and receiving), and $100 \%$ as in the remaining subaxes of the analysis form (individual defenseOne-to-one defense). The method of "TestRetest" was used with an interval of ten days of application, to calculate the reliability factor of the items of the defensive movements described in the sub-axes. The items reliability coefficient ranged from 0.88 to 0.94 and this indicates the high reliability factor of the form. The objectivity coefficients indicate a high degree of agreement among the peer reviewers that equalizes (1) as a correlation coefficient in most analysis axes (between $0.94: 0.100$ ).

Applying the Form in Match Analysis (the basic study):
The basic study was applied (analyzing the complex defensive performances) in the period from 20/9/2009 to 25/12/2009 utilizing a standardized form to analyze the matches ( Croatia vs Korea), (Spain vs Croatia) in the preliminary round, ( France vs Denmark), ( Croatia vs Poland) in the semi final round, and (Denmark vs Poland) to determine the winners of the third and fourth positions, and (France vs Croatia) to determine the winners of the first and second position.

\section{Results:}

The percentage of complex defensive movements in playing positions in the formation (5:1):

According to the quantitative analysis of the defensive movements for the different playing positions in the formation (5:1), figure (1) indicates the increasing role of the defensive movements of the midfield position, where the rate of such movements in the analyzed matches was $24.63 \%$ for the center back, 23.76 $\%$ for center forward, while the rate of the two backs follows in the second rank, concerning movements, as the left back got $21.48 \%$, and the right back got $16.84 \%$ of the total defensive movements. As for the right wing, this position got $8.41 \%$, while the left wing got only $5.20 \%$, and this may confirm the importance of the center - back positions in the successful performance of the formation (5:1). 


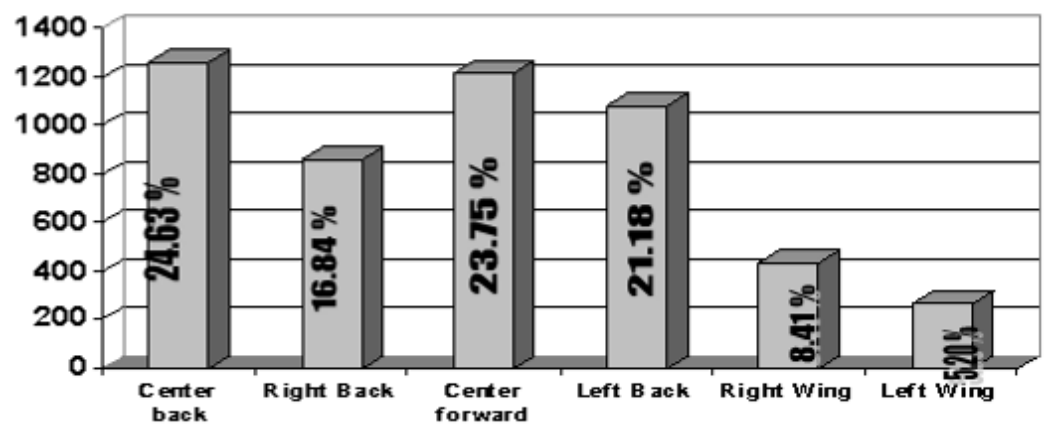

Indications of correlation of the successful movements between the playing positions in the formation $(5: 1)$ :

Figure (2) shows a significant correlation at the level 0.01 between the number of successful movements of the center forward position with the center back, and the two back players, and the number of successful movements of the center back with the right back, and the number of successful movements between the right back and the right wing, and the left back and the left wing, too.

The correlation was significant at the level 0.05 between the center back and the left back, and the center back and the left wing, whereas the movements were not significant in the remaining playing positions.

Figure 2

the significant correlation of the number of successful Defensive movements between playing positions

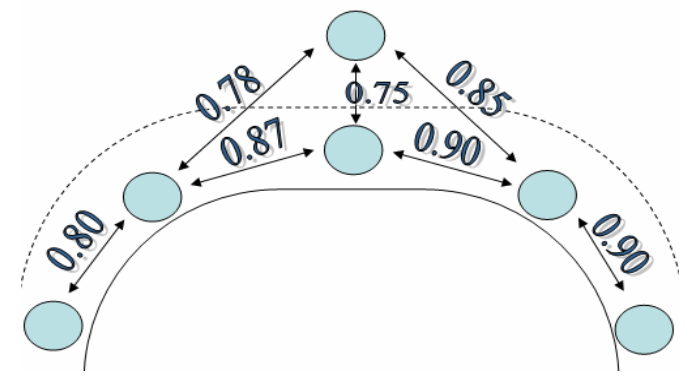

The quantitative analysis and the success percentage of the most common defensive movements of the playing positions in the formation (5:1) of the French national team (individual tactics):

Table (2) indicates the diversity of the complex individual defensive movements in the formation (5:1). Most of the movements were those of the two midfield positions (center back/ center forward) according to the nature of the attack (which comes in front of the goal). The movements of the center back to the side then making a block wall upward, then moving backward, were the most successful movements of the French national team, with a percentage of $89 \%$. This was repeated in two matches 16 times, taking into consideration the fact that during the analysis, 30 movements were recorded (15 of which were mentioned as the most common). As for the defensive movements of the left back, moving towards the side, then stopping to attack on the centre circle, and blocking the follow up movement after screening, represent the highest performance repetitions. Here, this movement was repeated 10 times successfully in two matches, and achieved a success rate of $83.5 \%$, taking into consideration that there were 26 individual defensive movements in this position. In the position of the left wing, the repetitions were equal for some movements, with 8 successful repetitions but moving to the side and stopping to attack and block a shot, achieved a success rate of $89 \%$ (10 individual defensive movements were recorded). The 
movements of the center forward varied and represented 30 movements that were recorded during the analysis. The table shows more than 15 of the most common movements: the commonest was the "diagonal defensive movement forward, then stops to attack or check" rated $87.5 \%$. As for the movements of the players in the back position, the repetition as stated in the table was 8 times, except for the "movement of avoiding blocking, then moving forward and stopping to attack" that was repeated 9 times, taking into consideration, that in addition to the movements mentioned in the table, (as the most common movements), there were more 15 additional movements recorded.

As for the position of right wing, the movement of "moving to a side and screening the cutting attacker", achieved $100 \%$ of 8 repetitions (note that there were 10 defensive movements in this position, recorded, too).

Table (2)

Percentages of types of successful complex defensive movements of the French world champions.

\begin{tabular}{|c|c|c|c|c|c|c|}
\hline$\stackrel{0}{:}$ & No & $\begin{array}{c}\text { Types of Complex Defense } \\
\text { Movements }\end{array}$ & $\begin{array}{c}\text { Total } \\
\text { movements }\end{array}$ & $\begin{array}{l}\text { Total } \\
\text { successful } \\
\text { repetitions }\end{array}$ & $\begin{array}{l}\text { Total } \\
\text { failing } \\
\text { repetitions } \\
\text { of French } \\
\text { players }\end{array}$ & $\begin{array}{l}\text { Percentage } \\
\text { of } \\
\text { successful } \\
\text { movements } \\
\text { of French } \\
\text { players }\end{array}$ \\
\hline \multirow{10}{*}{ 華 } & 1 & $\begin{array}{l}\text { Moving aside, then wall upwards and } \\
\text { moving back }\end{array}$ & 18 & 16 & 2 & 88.8 \\
\hline & 2 & $\begin{array}{l}\text { Moving aside, stopping to attack } \\
\text { opposing shooting with a wall }\end{array}$ & 14 & 12 & 2 & 85.7 \\
\hline & 3 & $\begin{array}{l}\text { Moving aside, defending by } \\
\text { obstructing after receiving the ball }\end{array}$ & 13 & 11 & 2 & 84.6 \\
\hline & 4 & $\begin{array}{l}\text { Moving aside, opposing to obstruct } \\
\text { attacker, defense against follow up } \\
\text { after obstructing }\end{array}$ & 11 & 9 & 2 & 81.8 \\
\hline & 5 & $\begin{array}{l}\text { Attacking, moving backwards and } \\
\text { opposing follow up after Obstruction }\end{array}$ & 11 & 9 & 2 & 81.8 \\
\hline & 6 & $\begin{array}{l}\text { Avoiding obstruction, diagonal defense } \\
\text { movement forward and stopping to } \\
\text { attack and fake opposition }\end{array}$ & 11 & 9 & 2 & 81.8 \\
\hline & 7 & $\begin{array}{l}\text { Avoiding obstruction, diagonal defense } \\
\text { movement aside, and stopping to } \\
\text { make a wall and oppose shooting }\end{array}$ & 10 & 9 & 1 & 90 \\
\hline & 8 & $\begin{array}{l}\text { Avoiding obstruction, moving aside } \\
\text { then diagonal defense movement } \\
\text { forward while attacking, tackling and } \\
\text { opposing obstruction }\end{array}$ & 10 & 9 & 1 & 90 \\
\hline & 9 & $\begin{array}{l}\text { Moving forward to attack and oppose } \\
\text { shooting the diagonal defense } \\
\text { backward }\end{array}$ & 11 & 9 & 2 & 88.8 \\
\hline & 10 & $\begin{array}{l}\text { Moving aside, obstructing a back } \\
\text { passing to follow up after Obstruction }\end{array}$ & 10 & 8 & 2 & 80 \\
\hline
\end{tabular}




\begin{tabular}{|c|c|c|c|c|c|c|}
\hline 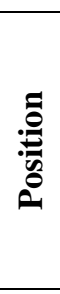 & No & $\begin{array}{c}\text { Types of Complex Defense } \\
\text { Movements }\end{array}$ & $\begin{array}{c}\text { Total } \\
\text { movements }\end{array}$ & $\begin{array}{l}\text { Total } \\
\text { successful } \\
\text { repetitions }\end{array}$ & $\begin{array}{l}\text { Total } \\
\text { failing } \\
\text { repetitions } \\
\text { of French } \\
\text { players }\end{array}$ & $\begin{array}{l}\text { Percentage } \\
\text { of } \\
\text { successful } \\
\text { movements } \\
\text { of French } \\
\text { players }\end{array}$ \\
\hline & 11 & $\begin{array}{l}\text { Avoiding obstruction, diagonal defense } \\
\text { movement and stopping to attack }\end{array}$ & 9 & 8 & 1 & 88.8 \\
\hline & 12 & $\begin{array}{l}\text { Diagonal defense movement forward } \\
\text { then backward then opposing with } \\
\text { wall to obstruct shooting by jumping }\end{array}$ & 9 & 8 & 1 & 88.8 \\
\hline & 13 & $\begin{array}{l}\text { Diagonal defense movement backward } \\
\text { then opposing to obstruct and follow } \\
\text { up after obstruction }\end{array}$ & 9 & 8 & 1 & 88.8 \\
\hline & 14 & $\begin{array}{l}\text { Moving aside, obstructing a passing } \\
\text { and defend by follow up after } \\
\text { obstruction }\end{array}$ & 9 & 8 & 1 & 88.8 \\
\hline & 15 & $\begin{array}{l}\text { Moving aside, stopping and attacking } \\
\text { and opposing the follow up after } \\
\text { obstruction }\end{array}$ & 10 & 8 & 2 & 80 \\
\hline \multirow{10}{*}{ 苞 } & 1 & $\begin{array}{l}\text { Moving aside, stopping to attack on the } \\
\text { circle and opposing the follow up after } \\
\text { obstruction }\end{array}$ & 12 & 10 & 2 & 83.3 \\
\hline & 2 & $\begin{array}{l}\text { Diagonal defense movement forward } \\
\text { then backward and defending by } \\
\text { obstructing after receiving }\end{array}$ & 11 & 9 & 2 & 88.8 \\
\hline & 3 & $\begin{array}{l}\text { Diagonal defense movement forward } \\
\text { then backward then opposing fake } \\
\text { movements }\end{array}$ & 11 & 9 & 2 & 88.8 \\
\hline & 4 & $\begin{array}{l}\text { Moving aside, stopping and attacking } \\
\text { by Diagonal defense movement } \\
\text { forward and opposing the shooting }\end{array}$ & 11 & 9 & 2 & 88.8 \\
\hline & 5 & $\begin{array}{l}\text { Moving aside, stopping while attacking } \\
\text { and opposing fake Movements }\end{array}$ & 10 & 8 & 2 & 80 \\
\hline & 6 & $\begin{array}{l}\text { Diagonal defense movement backward } \\
\text { and attacking while pushing out }\end{array}$ & 8 & 8 & - & 100 \\
\hline & 7 & $\begin{array}{l}\text { Moving aside and stopping with an } \\
\text { obstruction wall upwards }\end{array}$ & 10 & 8 & 2 & 80 \\
\hline & 8 & $\begin{array}{l}\text { Moving aside and opposing follow up } \\
\text { movement after obstruction and } \\
\text { receiving }\end{array}$ & 10 & 8 & 2 & 80 \\
\hline & 9 & $\begin{array}{l}\text { Avoiding obstruction and Diagonal } \\
\text { defense movement forward and } \\
\text { stopping to attack and opposing fake } \\
\text { movements }\end{array}$ & 10 & 8 & 2 & 80 \\
\hline & 10 & $\begin{array}{l}\text { Diagonal defense movement forward } \\
\text { then backward then avoiding } \\
\text { obstruction while attacking and } \\
\text { opposing fake movement }\end{array}$ & 10 & 8 & 2 & 80 \\
\hline
\end{tabular}




\begin{tabular}{|c|c|c|c|c|c|c|}
\hline 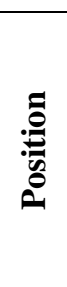 & No & $\begin{array}{c}\text { Types of Complex Defense } \\
\text { Movements }\end{array}$ & $\begin{array}{c}\text { Total } \\
\text { movements }\end{array}$ & $\begin{array}{l}\text { Total } \\
\text { successful } \\
\text { repetitions }\end{array}$ & $\begin{array}{l}\text { Total } \\
\text { failing } \\
\text { repetitions } \\
\text { of French } \\
\text { players }\end{array}$ & $\begin{array}{l}\text { Percentage } \\
\text { of } \\
\text { successful } \\
\text { movements } \\
\text { of French } \\
\text { players }\end{array}$ \\
\hline \multirow{8}{*}{ 离 } & 1 & $\begin{array}{l}\text { Moving aside then attacking and } \\
\text { opposing fake movement while } \\
\text { pushing out }\end{array}$ & 10 & 8 & 2 & 80 \\
\hline & 2 & $\begin{array}{l}\text { Moving aside, attacking and } \\
\text { obstructing the passing }\end{array}$ & 10 & 8 & 2 & 80 \\
\hline & 3 & $\begin{array}{l}\text { Moving aside, stopping while } \\
\text { attacking and opposing the Shooting }\end{array}$ & 9 & 8 & 1 & 88.8 \\
\hline & 4 & $\begin{array}{l}\text { Moving aside stopping with an } \\
\text { obstruction wall upward and pushing } \\
\text { off court }\end{array}$ & 10 & 8 & 2 & 80 \\
\hline & 5 & $\begin{array}{l}\text { Moving aside, attacking and opposing } \\
\text { dribbling }\end{array}$ & 8 & 6 & 2 & 75 \\
\hline & 6 & $\begin{array}{l}\text { Moving forward on the circle, stopping } \\
\text { and attacking, obstructing the passing } \\
\text { and opposing the shooting by jumping }\end{array}$ & 6 & 6 & - & 100 \\
\hline & 7 & $\begin{array}{l}\text { Moving aside then outwards while } \\
\text { attacking and opposing fake Movement }\end{array}$ & 8 & 6 & 2 & 75 \\
\hline & 8 & $\begin{array}{l}\text { Diagonal defense movement forward, } \\
\text { stopping to attack and oppose the } \\
\text { shooting }\end{array}$ & 10 & 6 & 2 & 60 \\
\hline \multirow{8}{*}{ 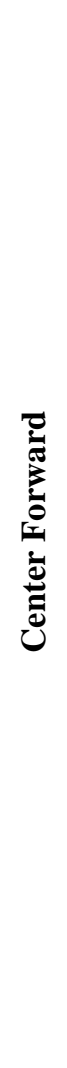 } & 1 & $\begin{array}{l}\text { Diagonal defense movement forward, } \\
\text { stopping to attack or Tackle }\end{array}$ & 16 & 14 & 2 & 87.5 \\
\hline & 2 & $\begin{array}{l}\text { Diagonal defense movement forward } \\
\text { then backward }\end{array}$ & 12 & 12 & Zero & 100 \\
\hline & 3 & $\begin{array}{l}\text { Attacking while tackling and opposing } \\
\text { to fake and diagonal movement } \\
\text { backwards }\end{array}$ & 14 & 12 & 2 & 85.7 \\
\hline & 4 & $\begin{array}{l}\text { Moving aside and thrust forward, } \\
\text { stopping to attack, tackle and obstruct } \\
\text { the passing }\end{array}$ & 13 & 12 & 1 & 92.3 \\
\hline & 5 & $\begin{array}{l}\text { Moving aside and stopping and } \\
\text { attacking while opposing fake } \\
\text { movement then pushing off court }\end{array}$ & 14 & 12 & 2 & 85.7 \\
\hline & 6 & $\begin{array}{l}\text { Moving aside and stopping and } \\
\text { forming a wall of opposition Upwards }\end{array}$ & 13 & 12 & 1 & 92.3 \\
\hline & 7 & $\begin{array}{l}\text { diagonal movement backwards and } \\
\text { stopping to attack and obstruct the } \\
\text { passing }\end{array}$ & 16 & 12 & 4 & 75 \\
\hline & 8 & $\begin{array}{l}\text { Diagonal defense movement forward } \\
\text { then aside while attacking and pushing } \\
\text { off court }\end{array}$ & 13 & 12 & 1 & 92.3 \\
\hline
\end{tabular}




\begin{tabular}{|c|c|c|c|c|c|c|}
\hline 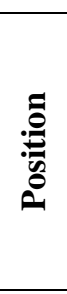 & No & $\begin{array}{c}\text { Types of Complex Defense } \\
\text { Movements }\end{array}$ & $\begin{array}{c}\text { Total } \\
\text { movements }\end{array}$ & $\begin{array}{c}\text { Total } \\
\text { successful } \\
\text { repetitions }\end{array}$ & $\begin{array}{l}\text { Total } \\
\text { failing } \\
\text { repetitions } \\
\text { of French } \\
\text { players }\end{array}$ & $\begin{array}{l}\text { Percentage } \\
\text { of } \\
\text { successful } \\
\text { movements } \\
\text { of French } \\
\text { players }\end{array}$ \\
\hline & 9 & $\begin{array}{l}\text { movement forward and backward then } \\
\text { blocking wall }\end{array}$ & 12 & 10 & 2 & 83.3 \\
\hline & 10 & $\begin{array}{l}\text { Moving aside then forward while } \\
\text { stopping to attack and oppose fake } \\
\text { movement }\end{array}$ & 12 & 10 & 2 & 83.3 \\
\hline & 11 & $\begin{array}{l}\text { Avoiding obstruction and diagonal } \\
\text { defense movement backwards and } \\
\text { stopping to attack and oppose fake } \\
\text { movement }\end{array}$ & 11 & 10 & 1 & 90.9 \\
\hline & 12 & $\begin{array}{l}\text { diagonal defense movement forwards, } \\
\text { then attacking with side movement } \\
\text { and obstructing the passing }\end{array}$ & 12 & 10 & 2 & 83.3 \\
\hline & 13 & $\begin{array}{l}\text { Moving aside then backward defense } \\
\text { movement and obstructing the passing }\end{array}$ & 12 & 10 & 2 & 83.3 \\
\hline & 14 & $\begin{array}{l}\text { Diagonal defense movement forwards, } \\
\text { then backwards and stopping to attack } \\
\text { and obstruct the passing }\end{array}$ & 10 & 9 & 1 & 90 \\
\hline & 15 & $\begin{array}{l}\text { Movement forward then stopping then } \\
\text { backwards and forming a blocking } \\
\text { wall upwards }\end{array}$ & 10 & 9 & 1 & 90 \\
\hline \multirow{8}{*}{ 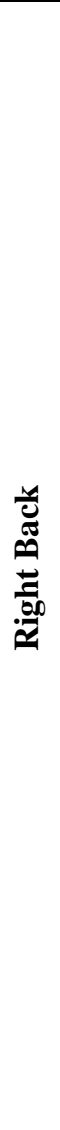 } & 1 & $\begin{array}{l}\text { Avoiding obstruction then diagonal } \\
\text { defense forwards and stopping to } \\
\text { attack }\end{array}$ & 11 & 9 & 2 & 81.8 \\
\hline & 2 & $\begin{array}{l}\text { diagonal defense movement forwards } \\
\text { then backwards and stopping to attack } \\
\text { tackle a blocking wall upwards }\end{array}$ & 10 & 8 & 2 & 80 \\
\hline & $\mathbf{3}$ & $\begin{array}{l}\text { Moving aside then defending through } \\
\text { follow up after Obstruction }\end{array}$ & 9 & 8 & 1 & 88.8 \\
\hline & 4 & $\begin{array}{l}\text { Moving forward and stopping while } \\
\text { attacking, tackling and opposing fake } \\
\text { movement }\end{array}$ & 9 & 8 & 1 & 88.8 \\
\hline & 5 & $\begin{array}{l}\text { Attacking, tackling and pushing off } \\
\text { court then diagonal defense backwards }\end{array}$ & 9 & 8 & 1 & 88.8 \\
\hline & 6 & $\begin{array}{l}\text { Moving aside, then attacking and } \\
\text { opposing fake movement }\end{array}$ & 15 & 8 & 7 & 53.3 \\
\hline & 7 & $\begin{array}{l}\text { Avoiding obstruction, then moving } \\
\text { aside then defending through follow up } \\
\text { after obstruction }\end{array}$ & 10 & 8 & 2 & 80 \\
\hline & 8 & $\begin{array}{l}\text { Diagonal defense movement forwards, } \\
\text { then } \\
\text { backwards and stopping to attack and } \\
\text { obstruct the passing }\end{array}$ & 9 & 8 & 1 & 88.8 \\
\hline
\end{tabular}




\begin{tabular}{|c|c|c|c|c|c|c|}
\hline 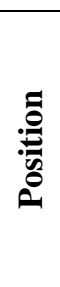 & No & $\begin{array}{c}\text { Types of Complex Defense } \\
\text { Movements }\end{array}$ & $\begin{array}{c}\text { Total } \\
\text { movements }\end{array}$ & $\begin{array}{l}\text { Total } \\
\text { successful } \\
\text { repetitions }\end{array}$ & $\begin{array}{l}\text { Total } \\
\text { failing } \\
\text { repetitions } \\
\text { of French } \\
\text { players }\end{array}$ & $\begin{array}{l}\text { Percentage } \\
\text { of } \\
\text { successful } \\
\text { movements } \\
\text { of French } \\
\text { players }\end{array}$ \\
\hline & 9 & $\begin{array}{l}\text { Avoiding obstruction then defending } \\
\text { through follow up after Obstruction }\end{array}$ & 10 & 8 & 2 & 80 \\
\hline & 10 & $\begin{array}{l}\text { diagonal defense movement forwards, } \\
\text { then backwards and attacking with a } \\
\text { blocking wall upwards }\end{array}$ & 9 & 8 & 1 & 88.8 \\
\hline \multirow{8}{*}{ 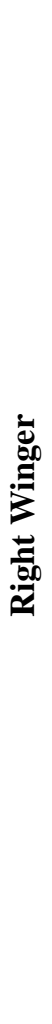 } & 1 & $\begin{array}{l}\text { Moving aside then defending through } \\
\text { follow up after Obstruction }\end{array}$ & 9 & 8 & 1 & 88.8 \\
\hline & 2 & $\begin{array}{l}\text { Moving aside while obstructing cutter } \\
\text { player }\end{array}$ & 8 & 8 & - & 100 \\
\hline & $\mathbf{3}$ & $\begin{array}{l}\text { Moving aside and opposing obstruction } \\
\text { then defending through follow up after } \\
\text { obstruction }\end{array}$ & 16 & 8 & 2 & 50 \\
\hline & 4 & $\begin{array}{l}\text { Moving aside then attacking with a } \\
\text { blocking wall }\end{array}$ & 10 & 8 & 2 & 80 \\
\hline & 5 & $\begin{array}{l}\text { Diagonal defense movement forward } \\
\text { then stopping to attack and obstruct the } \\
\text { passing }\end{array}$ & 10 & 8 & 2 & 80 \\
\hline & 6 & $\begin{array}{l}\text { Moving aside then stopping while } \\
\text { attacking and opposing fake Movement }\end{array}$ & 7 & 7 & - & 100 \\
\hline & 7 & $\begin{array}{l}\text { Moving aside then attacking and } \\
\text { opposing the shooting }\end{array}$ & 10 & 7 & 3 & 70 \\
\hline & 8 & $\begin{array}{l}\text { Moving aside then turning round and } \\
\text { moving forward then Attacking }\end{array}$ & 6 & 6 & 3- & 100 \\
\hline
\end{tabular}

The types of complex defense movements during defense based on tactical formations of One-to-one, delivery and receiving for each playing position, in the defense formation ( 5:1), and the synchronous defensive movements of the other playing positions:

The tables (3:7) show the synchronization between the movements of the playing positions and other defensive movements of neighboring playing positions, whether by Oneto-one or delivery and receiving methods.
Table 3, for example, shows that moving aside then forward then defending through follow up after obstruction synchronizes with moving aside then forwards then backwards then blocking wall by the center back player through One-to-one defense, as a condition for the integration between the defensive roles of players in the two center positions. 
Types of Complex Defense Movements of the Center Forward Position and the Synchronizing Movements by the Center

Back, Right Back and Left Back in the French National Team

\begin{tabular}{|c|c|c|c|c|}
\hline \multicolumn{2}{|c|}{$\begin{array}{l}\text { correlation with playing } \\
\text { positions on defense axes }\end{array}$} & Center Back & Right Back & Left Back \\
\hline $\begin{array}{l}\text { Center } \\
\text { Forward }\end{array}$ & $\begin{array}{l}\text { One-to-one } \\
\text { defense }\end{array}$ & $\begin{array}{l}\text { - diagonal defense } \\
\text { movement forward, then } \\
\text { backward and stopping to } \\
\text { attack and oppose fake } \\
\text { movement } \\
\text { * moving aside then } \\
\text { forward then backward } \\
\text { then defense through } \\
\text { follow up after obstruction } \\
\text { - moving aside then } \\
\text { thrusting forward and } \\
\text { stopping to attack, tackle } \\
\text { and obstruct passing } \\
* \text { moving aside then } \\
\text { obstructing the passing and } \\
\text { defend through follow up } \\
\text { after obstruction and } \\
\text { receiving the ball } \\
\text { - moving forward then } \\
\text { backward avoiding } \\
\text { obstruction and performing } \\
\text { a blocking wall } \\
* \text { moving forward then } \\
\text { backward then moving } \\
\text { aside and defending } \\
\text { through obstruction }\end{array}$ & $\begin{array}{l}\text { - Moving aside } \\
\text { then forward, } \\
\text { stopping to attack } \\
\text { and oppose fake } \\
\text { movements then } \\
\text { passing. } \\
\text { * Moving aside } \\
\text { then stopping and } \\
\text { forming a wall to } \\
\text { oppose the shooting } \\
\text { by jumping }\end{array}$ & $\begin{array}{l}\text { - Moving aside then } \\
\text { stopping while } \\
\text { attacking and opposing } \\
\text { fake movements then } \\
\text { pushing off court } \\
\text { * Moving aside, then } \\
\text { stopping while } \\
\text { attacking, then } \\
\text { diagonal defense } \\
\text { backward and } \\
\text { opposing the shooting }\end{array}$ \\
\hline $\begin{array}{l}\text { Center } \\
\text { Forward }\end{array}$ & $\begin{array}{l}\text { Delivery } \\
\text { and } \\
\text { receiving } \\
\text { defense }\end{array}$ & $\begin{array}{l}\text { - moving forward then } \\
\text { backward avoiding } \\
\text { obstruction and defending } \\
\text { through follow up after } \\
\text { obstruction. } \\
* \text { moving aside then } \\
\text { stopping to attack and } \\
\text { oppose the shooting with a } \\
\text { wall }\end{array}$ & $\begin{array}{l}\text { - Attacking, } \\
\text { tackling and } \\
\text { opposing fake } \\
\text { movements, with } \\
\text { an diagonal } \\
\text { movement } \\
\text { backward. } \\
\text { * Moving aside } \\
\text { then stopping } \\
\text { forming a blocking } \\
\text { wall to oppose } \\
\text { shooting by } \\
\text { jumping high. } \\
\\
\text { - Diagonal } \\
\text { movement } \\
\text { backward and } \\
\text { stopping to attack } \\
\text { and obstructing the }\end{array}$ & $\begin{array}{l}\text { - diagonal defense } \\
\text { movement forward, } \\
\text { then backward and } \\
\text { stopping to attack and } \\
\text { obstructing the passing } \\
* \text { diagonal defense } \\
\text { movement forward, } \\
\text { then stopping to attack } \\
\text { and opposing the } \\
\text { shooting. }\end{array}$ \\
\hline
\end{tabular}




\begin{tabular}{|c|c|c|c|}
\hline $\begin{array}{l}\text { correlation with playing } \\
\text { positions on defense axes }\end{array}$ & Center Back & Right Back & Left Back \\
\hline & & $\begin{array}{l}\text { passing } \\
\text { *Moving forward } \\
\text { then stopping, } \\
\text { attacking and } \\
\text { opposing fake } \\
\text { movement }\end{array}$ & \\
\hline
\end{tabular}

- The center forward player defense movements

* The dynamic correlation of the complex defense movements in the playing positions described in the table

Table 4

Type of Complex Defense Movements of the Center Back Position and the Synchronizing Movements by the Center Forward, Right Back and Left Back in the French National Team

\begin{tabular}{|c|c|c|c|c|}
\hline \multicolumn{2}{|c|}{$\begin{array}{c}\text { correlation with } \\
\text { playing positions on } \\
\text { defense axes }\end{array}$} & $\begin{array}{c}\text { Center Back Center } \\
\text { Forward }\end{array}$ & Right Back & Left Back \\
\hline $\begin{array}{c}\text { Center } \\
\text { Back }\end{array}$ & $\begin{array}{l}\text { One-to- } \\
\text { one } \\
\text { defense }\end{array}$ & $\begin{array}{l}\text { - moving aside then } \\
\text { stopping to attack and } \\
\text { oppose } \\
\text { to obstruct attacker. } \\
\text { * moving forward } \\
\text { then backward avoiding } \\
\text { obstruction, then } \\
\text { Performing a blocking } \\
\text { wall. } \\
\text { - Moving aside, } \\
\text { then stopping and } \\
\text { opposing to obstruct } \\
\text { attacker. } \\
\text { * Attacking, tackling and } \\
\text { opposing fake movement, } \\
\text { with } \\
\text { diagonal movement } \\
\text { backward. } \\
\text { - Moving aside then } \\
\text { diagonally forward and } \\
\text { opposing to screen an } \\
\text { attacker } \\
* \text { Moving forward then } \\
\text { backward then forming a } \\
\text { blocking wall upwards. } \\
\text { - Moving aside then } \\
\text { opposing to obstruct } \\
\text { attacker and defending } \\
\text { against follow up after } \\
\text { obstruction. } \\
\text { * Moving forward then } \\
\text { backward avoiding }\end{array}$ & $\begin{array}{l}\text { - Moving aside then } \\
\text { defending through } \\
\text { obstruction after receiving } \\
\text { the ball. } \\
\text { * Moving aside then } \\
\text { diagonal defense } \\
\text { movement while } \\
\text { attacking with a blocking } \\
\text { wall. } \\
\text { - Moving forward and } \\
\text { backward then to the side } \\
\text { and defending through } \\
\text { follow up after } \\
\text { obstruction. } \\
\text { * Moving forward and } \\
\text { stopping without } \\
\text { attacking and opposing } \\
\text { fake movement } \\
\text { - Moving aside and } \\
\text { stopping while attacking } \\
\text { and defending Through } \\
\text { obstruction after receiving } \\
\text { the ball. } \\
\text { * Moving aside and } \\
\text { stopping while attacking } \\
\text { and pushing off court. } \\
\text { - Moving aside then } \\
\text { stopping while attacking } \\
\text { and pushing off court. } \\
\text { * Moving aside then } \\
\text { defending through } \\
\text { obstruction and follow up }\end{array}$ & $\begin{array}{l}\text { Moving aside then } \\
\text { opposing to screen } \\
\text { attacker and defending } \\
\text { against follow up after } \\
\text { obstruction. } \\
\text { * Moving aside then } \\
\text { stopping and forming a } \\
\text { blocking wall upwards } \\
\text { - Moving aside then } \\
\text { opposing follow up after } \\
\text { obstruction and receiving. } \\
\text { * Avoiding obstruction } \\
\text { then attacking and } \\
\text { opposing fake movement. } \\
\text { - Moving aside then } \\
\text { avoiding obstruction then } \\
\text { attacking with a blocking } \\
\text { wall. } \\
\text { * Moving aside then } \\
\text { opposing follow up } \\
\text { movement after } \\
\text { obstruction and receiving. } \\
\text {-Moving aside then } \\
\text { stopping to attack and } \\
\text { opposing the shooting } \\
\text { with a blocking wall. } \\
* \text { Moving aside then } \\
\text { stopping to attack on the } \\
\text { circle and opposing the } \\
\text { follow up movement after } \\
\text { obstruction. }\end{array}$ \\
\hline
\end{tabular}




\begin{tabular}{|c|c|c|c|}
\hline $\begin{array}{l}\text { correlation with } \\
\text { playing positions on } \\
\text { defense axes }\end{array}$ & $\begin{array}{c}\text { Center Back Center } \\
\text { Forward }\end{array}$ & Right Back & Left Back \\
\hline & $\begin{array}{l}\text { obstruction and forming a } \\
\text { blocking wall. } \\
\text { - Moving aside then } \\
\text { obstructing the back } \\
\text { passing to follow up after } \\
\text { obstruction } \\
\text { * Avoiding screening } \\
\text { then diagonal defense } \\
\text { movement backwards and } \\
\text { stopping to attack and } \\
\text { oppose fake movement. } \\
\text { - Avoiding screening then } \\
\text { diagonal defense } \\
\text { movement forward and } \\
\text { stopping to attack and } \\
\text { oppose fake movement. } \\
\text { * Moving backward then } \\
\text { moving aside and } \\
\text { opposing fake movement } \\
\text { - Moving aside then } \\
\text { attacking and opposing } \\
\text { dribbling } \\
\text { * Avoiding screening } \\
\text { then attacking and } \\
\text { obstructing the passing }\end{array}$ & $\begin{array}{l}\text { after obstruction } \\
\text { - Moving aside then } \\
\text { moving forward and } \\
\text { backward then forming a } \\
\text { blocking wall. } \\
\text { * Avoiding obstruction } \\
\text { then defending through } \\
\text { follow up after } \\
\text { obstruction }\end{array}$ & \\
\hline
\end{tabular}

- The center back player defense movements

* The dynamic correlation of the complex defense movements in the playing positions described in the table 
Table (5) shows that, in the defense performance of One-to-one and delivery and receiving methods, the side movements of the right back, then defending through the follow up movement after obstruction, synchronize with the defense movements of the center back, i.e., moving aside, then stopping, attacking and pushing off court

Table (5)

Types of the complex defense movements of the right back position and the synchronous movements of the center forward and the center

Back and the left wing of the French national team.

\begin{tabular}{|c|c|c|c|c|}
\hline \multicolumn{2}{|c|}{$\begin{array}{l}\text { correlation with } \\
\text { playing positions on } \\
\text { defense axes }\end{array}$} & $\begin{array}{c}\text { Center Back Center } \\
\text { Back }\end{array}$ & Center Forward & Right Wing \\
\hline 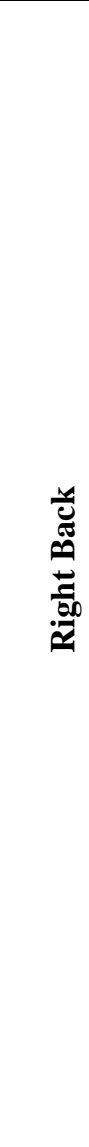 & 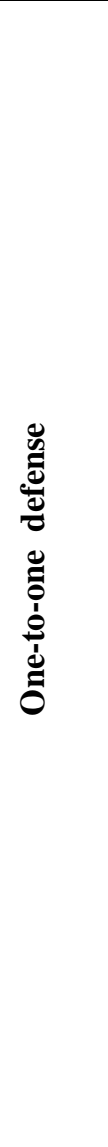 & $\begin{array}{l}\text { - diagonal defense } \\
\text { movement forward, then } \\
\text { stopping to attack and } \\
\text { oppose fake movement } \\
\text { * Moving aside then } \\
\text { defending through } \\
\text { obstruction after } \\
\text { receiving the ball } \\
\text { - Avoiding obstruction } \\
\text { then diagonal defense } \\
\text { movement forward and } \\
\text { stopping to attack ball. } \\
\text { * Moving forward and } \\
\text { backward then to the } \\
\text { side and defending } \\
\text { through follow up after } \\
\text { obstruction. } \\
\text { - Moving aside, then } \\
\text { attacking and opposing } \\
\text { fake movement } \\
* \text { Avoiding obstruction } \\
\text { then moving forward on } \\
\text { the circle and opposing } \\
\text { follow up after } \\
\text { obstruction }\end{array}$ & $\begin{array}{l}\text { - diagonal defense } \\
\text { movement forward, then } \\
\text { to the side while } \\
\text { attacking and opposing } \\
\text { fake movement } \\
\text { * moving aside then } \\
\text { stopping and attacking } \\
\text { while opposing the } \\
\text { shooting } \\
\text { - moving forward then } \\
\text { attacking and opposing } \\
\text { fake movement and the } \\
\text { shooting } \\
\text { * moving aside then } \\
\text { thrusting forwards and } \\
\text { stopping to attack and } \\
\text { obstruct the passing }\end{array}$ & $\begin{array}{l}\text { - Diagonal defense } \\
\text { movement forward then } \\
\text { backward } \\
\text { * Moving aside then } \\
\text { attacking with a blocking } \\
\text { wall upwards } \\
\text { - Diagonal defense } \\
\text { movement forward then } \\
\text { aside and obstructing the } \\
\text { passing } \\
\text { * Moving aside in then } \\
\text { out while attacking and } \\
\text { opposing fake } \\
\text { movement }\end{array}$ \\
\hline
\end{tabular}




\begin{tabular}{|c|c|c|c|}
\hline $\begin{array}{c}\text { correlation with } \\
\text { playing positions on } \\
\text { defense axes }\end{array}$ & $\begin{array}{c}\text { Center Back Center } \\
\text { Back }\end{array}$ & Center Forward & Right Wing \\
\hline 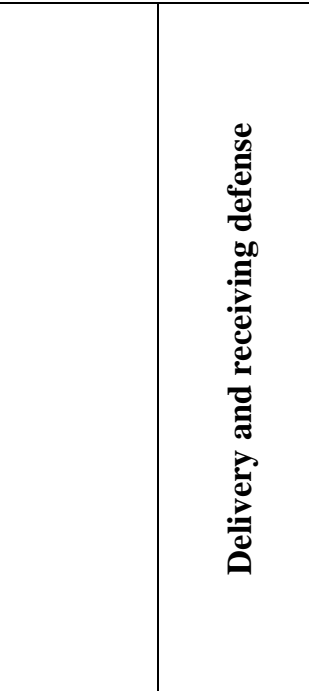 & $\begin{array}{l}\text { - Moving aside then } \\
\text { defending through } \\
\text { follow up after } \\
\text { obstruction. } \\
\text { * Moving aside then } \\
\text { stopping while attacking } \\
\text { and pushing off court } \\
\text { - Avoiding obstruction } \\
\text { then moving aside then } \\
\text { defending through } \\
\text { follow up after } \\
\text { obstruction } \\
\text { * Moving aside then } \\
\text { stopping while attacking } \\
\text { and pushing off court }\end{array}$ & $\begin{array}{l}\text { - Moving aside then } \\
\text { stopping to attack and } \\
\text { obstruct the passing } \\
* \text { Moving aside then } \\
\text { stopping to attack and } \\
\text { oppose fake movement } \\
\text { while moving aside }\end{array}$ & $\begin{array}{l}\text { - Moving aside then } \\
\text { attacking and obstructing } \\
\text { the passing while } \\
\text { pushing off court } \\
\text { * Moving aside then } \\
\text { attacking and opposing } \\
\text { fake movement }\end{array}$ \\
\hline
\end{tabular}

\section{- The center back player defense movements}

* The dynamic correlation of the complex defense movements in the playing positions described in the table

There is also a synchronous correlation in the movements between the players of the wing and back positions (as shown in table 7) because moving to the side, attacking and blocking the fake of the right wing, requires a movement to the side, stopping and making a block wall to block shooting by jumping high with the right back.

Table (6)

Types of the complex defense movements of the left back position and the synchronous movements of the center forward and the center back and the right wing of the French national team.

\begin{tabular}{|c|c|c|c|c|}
\hline \multicolumn{2}{|c|}{$\begin{array}{l}\text { correlation with playing } \\
\text { positions on defense axes }\end{array}$} & $\begin{array}{c}\text { Center Back Center } \\
\text { Back }\end{array}$ & Center Forward & Left Wing \\
\hline 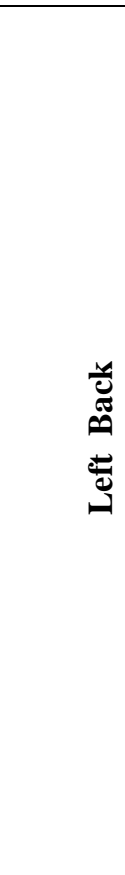 & 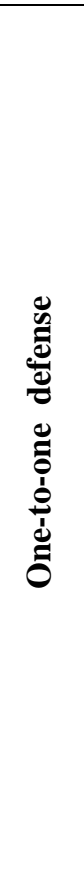 & $\begin{array}{l}\text { - Avoiding obstruction } \\
\text { then diagonal defense } \\
\text { movement forward and } \\
\text { stopping to attack and } \\
\text { oppose fake movement. } \\
\text { * Moving side and } \\
\text { obstructing the passing } \\
\text { and defending through } \\
\text { follow up after } \\
\text { obstruction. } \\
\text { - Diagonal defense } \\
\text { movement forward, then } \\
\text { backward while } \\
\text { avoiding obstruction } \\
\text { then attacking. } \\
* \text { Attacking, moving } \\
\text { backward and opposing } \\
\text { the follow up after } \\
\text { obstruction }\end{array}$ & $\begin{array}{l}\text { - Moving aside then } \\
\text { stopping and forming a } \\
\text { blocking wall upward. } \\
\text { * Moving aside then } \\
\text { attacking and tackling } \\
\text { and obstructing the } \\
\text { passing. } \\
\text { - Diagonal Defense } \\
\text { movement back to the } \\
\text { left then to the right } \\
\text { while attacking and } \\
\text { obstructing the passing. } \\
* \text { Diagonal movement } \\
\text { backward then stopping } \\
\text { to attack and obstruct the } \\
\text { passing }\end{array}$ & $\begin{array}{l}\text { - Diagonal defense } \\
\text { movement backward, } \\
\text { then stopping to attack } \\
\text { and pushing off court. } \\
* \text { Moving aside then } \\
\text { attacking while } \\
\text { opposing fake } \\
\text { movement }\end{array}$ \\
\hline
\end{tabular}




\begin{tabular}{|l|l|l|l|l|}
\hline \multicolumn{2}{|c|}{$\begin{array}{c}\text { correlation with playing } \\
\text { positions on defense axes }\end{array}$} & $\begin{array}{c}\text { Center Back Center } \\
\text { Back }\end{array}$ & Center Forward & Left Wing \\
\hline & & $\begin{array}{l}\text { - Moving aside then } \\
\text { stopping to attack on the } \\
\text { circle and opposing } \\
\text { follow up after } \\
\text { obstruction } \\
* \text { Avoiding obstruction }\end{array}$ & $\begin{array}{l}\text { - Diagonal defense } \\
\text { movement forward then } \\
\text { stopping to attack and } \\
\text { oppose fake movement } \\
* \text { Moving aside then } \\
\text { stopping and attacking } \\
\text { while opposing the } \\
\text { shooting then pushing } \\
\text { off court }\end{array}$ & $\begin{array}{l}\text { - Moving aside out then } \\
\text { in while attacking and } \\
\text { opposing the shooting } \\
\text { attacking and } \\
\text { obstructing the passing }\end{array}$ \\
& $\begin{array}{l}\text { then attacking and } \\
\text { opposing fake } \\
\text { movement }\end{array}$ & & \\
\hline
\end{tabular}

The center back player defense movements

* The dynamic correlation of the complex defense movements in the playing positions described in the table

Table (7)

Types of the complex defense movements of the two wingers

And the synchronous movements of the two backs

of the French national team.

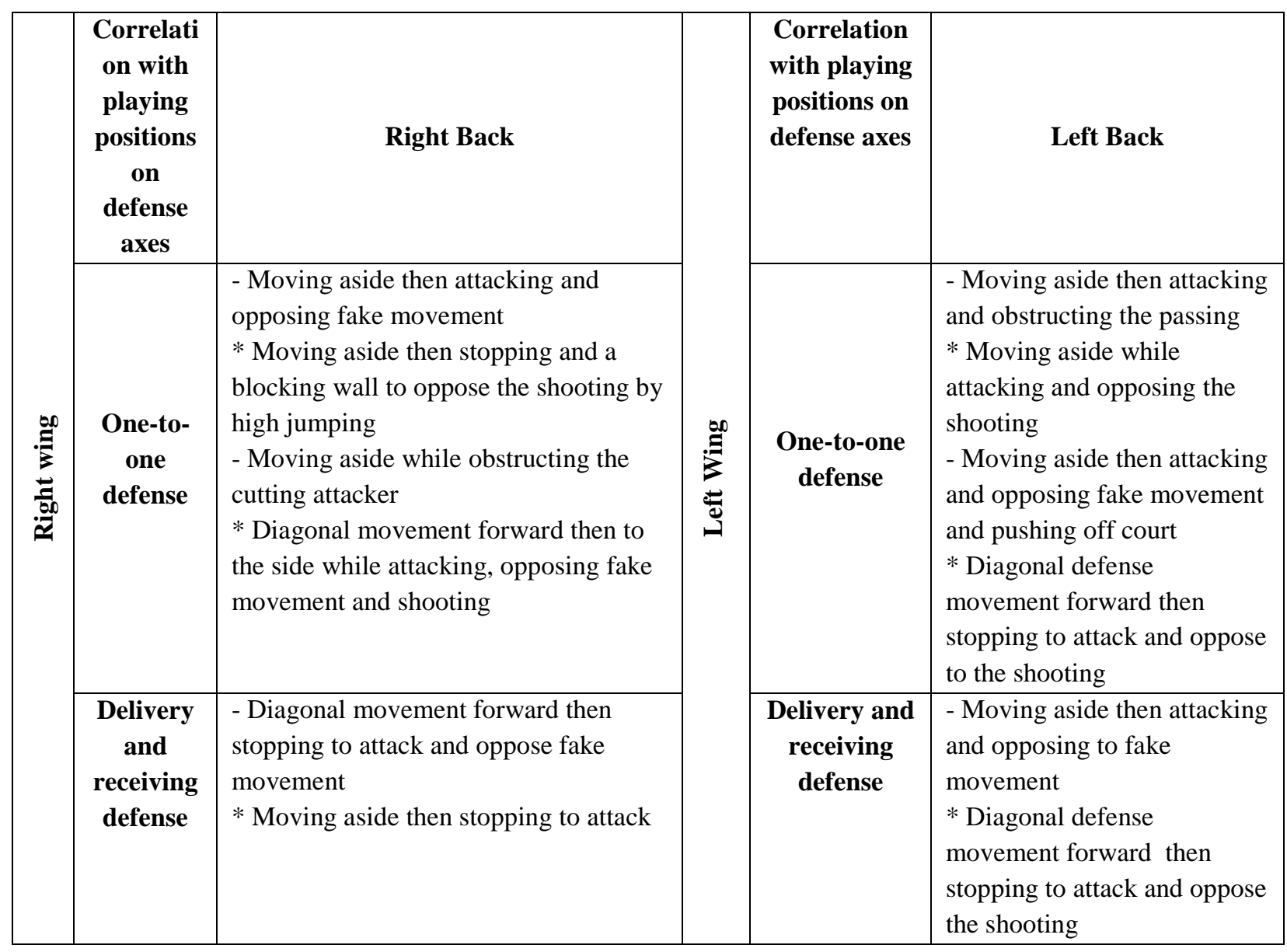

- The center back player defense movements

* The dynamic correlation of the complex defense movements in the playing positions described in the table

\section{Discussion:}


Comparing the quantitative analysis of the defensive performances in the formation $(5: 1)$ of the different playing positions according to the results of matches analysis in the research (see figure 1) and the outcomes of the analysis on the e-mail address of the International Handball Federation concerning the French national team after 10 matches, it is clear that there is compatibility between the rate of participation and roles of the playing positions for the first four teams in the championship (France, Croatia, Poland and Denmark) with the results of the analysis made by the international federation concerning the French team. The statistics indicate that the total attempts to score from the central zone are (298). Out of these, the attacking teams scored (125) goals constituting $42 \%$ (from the center court area and the two backs position), whereas the attempts of shooting from the wings positions were (45) in number out of which the competing teams could score (19) goals, constituting $42 \%$. The statistical comparison of the scoring attempts from the center court area and wings positions indicates that the percentage does not exceed $15 \%$ for the two wings, so that $85 \%$ of the score attempts on the French goal came from the middle area. This characterizes the defensive performances of the teams participating in the world championship, and it is compatible with the results of the French team as a model for the successful defensive performances which greatly contributed to winning the first rank. The percentage of successful attempts to block attacks was $58 \%$ of the total attempts. On the other hand, reaching the same analysis results reflects the reliability of the analysis form used in this research for the objective analysis of the types of complex defensive movements of the different playing positions in the formation (5:1).
The above mentioned percentages $-85 \%$ of the shooting attempts coming from the center court area - and the occurrence of different types of complex defensive movements in the individual defense of the playing positions (the center court area and the two backs), as 111 complex defensive movements were recorded for these positions, while only 20 movements were recorded for the positions of the two wings.

The performance of the center back (libero) which had 30 movements was attributed by the researcher to the source of attack of the competing teams attack. This agrees with the special case of this position, referred to by Molino (2003) as a distinguished position that needs high ability of expectation, covering, block wall, cutting passes, defensive help, screening on the attacker and the defense with follow up after screening. Thus, this position is considered the main one among others in the defensive process, and it should bear the responsibility of deciding the method of defensive performance during the organized defense.

The process of "impeding the passing" is one of the most important analysis results of the complex defensive movements of the individual defense tactics concerning the forward midfielder. This is often the last stage of some defensive performances, and it involves the ability of starting counter-attacks, but according to Ragab (2009), this requires a high speed reaction, and great ability of starting at a great speed, and the motor speed of complex defensive performances of the player, to enable him to make a quick start for counter-attacks.

The analysis (see table 2) illustrates the most important characteristics of the two back players of the French national team, concerning side movements, diagonal forward-backward movements, attacking and blocking of fake and shooting, besides avoiding screening then 
defense on the follow-up movements after screening. The researcher believes that this is related to the defensive task of the player, where he is considered a link between the neighboring playing positions of the wing and the back midfielder. $\mathrm{He}$, also, represents an important defensive axis as he was able, cooperating with the midfielder, to block a lot of scoring attempts, according to the statistics of the international federation concerning the French national team in Croatia championship (2009). The right back managed to stop 53 attempts (out of 81 , with a success rate of $65 \%$. As for the left back, he managed to stop 40 attempts (out of 63, with a success rate of 63.5 $\%)$.

Due to the space available for the wing player, and the correlation of his movements with the back players, most of his movements are to the side followed by attacking to block fake or attacking by a block wall. This is because the correlation strategy and complex defensive movements in the formation (5:1) depends mainly on the position of scoring attempt, and mutual and integrated cooperation between the neighboring playing positions, the main axis of the defense process can be judged according to the analysis results, see tables (3:7), as the two positions of the midfield, and their relation with the two neighboring positions( the two positions of the back players).The integrated team work, as Hammouda and Salem noted, (2008), needs exploitation of the operations that aim at correlating and understanding through mutual motor operations (One-to-one, or delivery and receiving) between the team members according to the playing situations and the movement of players in the real offensive performance in the match. By looking at table (3), we can say that moving by delivery and receiving between the back and forward midfielders is controlled by the offense strategy through the two play-makers and the pivot player. Thus, if the performance of the pivot player including moving to the side and diagonally forward while screening the defender (forward midfielder) coincides with the performance of the play-maker who gets the ball and then makes a double fake (left, right, left), then shooting with high jump, so, the defensive performance of the front midfielder is decided in " moving forward, then backward, avoiding screening ( from the pivot player), then the defense on the follow-up movement after screening" (that means he watches the pivot player), while the back midfielder watches the player who shoots ( the playmaker) by moving to the side, then stopping to attack and block shooting by an upward block wall”.

As for the tactical defense with the One-to-one method (see table 4), the performance of the back midfielder has a dynamic purposeful relation with right back to block the attempt to score made by the attacker (left back, and the pivot player) where the right back" moves to the side, then follows the diagonal defensive movement while attacking by a block wall" against the offensive performance of the left back who may fake without the ball, then he receives the ball and makes a complex fake action by shooting and then passing to the "pivot player" who cuts on the circle to screen against the back midfielder, to be able to receive the passing from the left back, and to stop his danger- as he is in a position of scoring- the back midfielder moves to the side and then he defends on screening after receiving the ball. Thus, the harmony of movements of the back midfielders and the two back players close the gaps in front of the attackers, and that means they should repeat the attempts and think of another tactic. This gives the defending team- as long as it did not score any goals- more chance to block the attack or to cut and take the ball. 
Analyzing the complex defensive actions of the two positions of the back and the wing (see table 6), we find that the major role is blocking the source of danger, and keeping the fake away from the midfield position (towards outer area). This gives the defending team the advantage of obstructing the attack and the potential of considering the negative playing a point against it. This also reduces the danger of the offensive action, i.e., if the right back is in possession of the ball, and performs his complex offensive role" receiving, then, making complex fake with rotation of the arm then passing to the rightwing". So, the defensive role of the left back is limited to "the back-diagonal defensive movements, then stopping to attack and pushing the wing off court", then the left winger completes the defensive role in the attacking process, and keeping away towards the outer area, and blocking the feint from the right winger who camouflages trying to shoot by complex action, " receiving, then camouflaging right- left- right, then shooting with jumping forward".

\section{Conclusion:}

-The qualitative and quantitative analysis of the complex defensive performance of the distinguished teams (e.g., France), allows us to identify the modern complex defensive movements, and their importance in blocking the attack. This is not concerning the level of individual defensive tactics only, but also the level of team defense, especially in the formation $(5: 1)$. The analysis, also, sets the foundations of defensive strategy, through the players' roles in the neighboring playing positions, where cooperation and understanding between these positions represent the "password" and the key to success in the group defensive performance.

-In the light of findings of the study, the researcher recommends to consider;
--The different types of the defensive movements that could be recorded through the analysis of the French national team performance: ( individual and group tactics) in the formation ( $5: 1$ ), as basic pillars upon which the process of tactical training of the beginners sector is to be built.

--This should happen applying the principles of the sports training, as gradual processes from the simplest steps to the complicated ones, to acquire these complex defensive performances and provide the training conditions that are similar to those in the matches. This can be achieved by linking the defensive situation with the accompanied offensive situation, taking into consideration the importance of understanding and cooperation, and keeping the minimum items of the successful defensive performance, particularly those concerning the time and speed of the achievement. This in turn will be reflected on the level of the players' adaptation, and their ability of enduring pressure and physical and skillful burdens related to the quality of achievements in handball.

\section{References}

1. Ali, A. (1998): An analytical study of some defensive and offensive variables of the Egyptian national handball team for men, unpublished MA Thesis, faculty of sport education for men, Cairo, Helwan University, P.P.10-17

2. Costa , J., Elzauaurdia, L. ( 2003 ) : Offence training system Tactic development of a play conception, top ANDEBOL , symposium for coaches, IHF , Portugal, (2) P. 118 .

3. Czerwinski, J. \& Taborsky, F. (1997): Basic Handball, Methods / tactics / technique .Avis - werbung, Austria P.P. $32-34$.

4. Dios, J. (2000): World Championship Egypt 99 analysis, Reflex ion ship and 
trends in handball starting from this event EHF. P.85

5. Feldman, K. (2002): Maintained Efforts against passive Matche Top ANDEBOL symposium for coaches $I H F$.Portugal. (2) P.3.

6. Feldman, K. (2003): Specific ally Training Rapid play, special supplement to world Handball Magazine, Portugal. (2). P.45.

7. Salem, G. (2002): Modern Handball (foundations and applications), first edition, Mansha'at EL-Ma'aref, Alexandria, P.P. 301-321.

8. Darwish, K., Abbas, I., Ali, S. (1998), The Physiological Bases of Handball Training (Theories and Applications), Markaz el-ketab Lel-Nashr, Cairo, (p. 44).

9. Darwish, K., Abbas, I., Ali, S. (1999): Defense in Handball, first edition, Markaz el-ketab Lel-Nashr, Cairo, (p. 42).

10. Darwish, K., Morsi, K., Abu Zeid, E. (2002): Measurement, Evaluation and Analyzing matches in handball (theoriesapplications), first edition, Markaz el-Ketab Lel-Nashr, Cairo, P. 56.

11. Klein, G. (1988): Selected aspects of players performance at the 1988 Men's $E G H$ in Italy EHF. P.83.

12. Ragab, M. (2009): Developing the speed of tactical defensive performances, unpublished PH.D thesis, Faculty of Sport Education for Men, Alexandria University, P. 96, P. 109.

13. Kamel, M. (2007): Dynamics of Change in the complex offensive skill performances during the matches of the last handball world championship "men" , the scientific Journal of Sport and Physical Education, Part 2, Faculty of Sport
Education for Girls, Alexandria university, issue no. 33, P.P. 73-77.

14. Kamel, M. (2008): Speed of complex offensive tactics performances of the African players and European players of handball for men, the Scientific Journal of sport and Physical Education, Part 2, Faculty of Sport Education for Girls, Alexandria University, issue no. 35, P.P. 2331.

15. Hammouda, K., Salem, G. (2008): Offense and Defense in Handball, first edition, Max Group Company, Alexandria. P.P.416-418.

16. Molino, A. (2003): Basic tactics in playing handball - Fiba a assist magazine.

(3) P.86.

17. Moneir Gerges (2004), Handball for all, Dar El-Fikr El-Arabi, Cairo, P. 249.

18. Pollany, W. (2000): Match result save all in the head a multi dimensional analysis of tows final Matches, IHF/EHF. P.86.

19. Taborsky, F. (1999): The Method of Quantities Evaluation of Match Performance in handball, special supplement to world Handball Magazine, Portugal. (1) P.88.

20. El-Katami, W. (2005): Planning a training programme for an effective development of some requirements of the defensive tactical performance against the fast break in handball, unpublished PH.D Thesis, Faculty of Sport Education for Girls, Alexandria University, P.P.10-13.

21. Dabour, Y. (2007): Analyzing the results of handball world cup championship, Germany 2007, in the light of effectively and productivity together, theories and applications, faculty of sport education for men, Alexandria University, issue no. 63, P.P. 83-85.

22. WWW.IHF.INFO 